\title{
Power Coupling Efficiency Enhancement in Multimode Step-Index Fiber Using Refractive and Diffractive Microlenses
}

\author{
S. Selvanandan ${ }^{1}$ and P. M. Anbarasan ${ }^{2}$ \\ ${ }^{1}$ Department of Physics, ACS College of Engineering, Bangalore 560 074, India \\ ${ }^{2}$ Department of Physics, Periyar University, Salem 636011 , India
}

Correspondence should be addressed to S. Selvanandan, selvanandan@gmail.com

Received 9 September 2010; Revised 19 November 2010; Accepted 5 December 2010

Academic Editor: Victor Arrizon

Copyright (C) 2010 S. Selvanandan and P. M. Anbarasan. This is an open access article distributed under the Creative Commons Attribution License, which permits unrestricted use, distribution, and reproduction in any medium, provided the original work is properly cited.

\begin{abstract}
A novel study has been undertaken for the coupling efficiency enhancement on planar (refractive and diffractive) microlenses as coupling elements with various geometrical shapes in fibre optics communication. When the dispersion of the planar diffractive microlens is expressed in terms of an Abbe number, it is easy to solve for the refractive and diffractive power coupling efficiency that result in a single material. Coupling loss is reduced with microlens used as coupler even when increasing wavelength of the Gaussian beam. A slight difference of power coupling efficiency is observed with the different direction of electric polarisation for a Gaussian beam of large spot size at its waist due to coupling property has been discussed and shown to agree well with theoretical model.
\end{abstract}

\section{Introduction}

The great advances made by optical telecommunications have brought about the industrial manufacturing of a whole set of components for optical fibres and for each component; there is much competition to optimise the performance while reducing cost. In designing optical communication systems, one of the greatest problems is to reduce the optical coupling loss from a laser diode to a fibre or from a fibre to the optical device or vice versa. Before connecting the fibre to the optical system, coupling characteristics such as coupling losses and alignment tolerances are investigated. Recent progress in optical technology and optical devices has made optical fibre transmission systems available for practical use $[1,2]$.

Effective power coupling between a laser diode and a fibre is a big concern in many applications. In optical fibre communications, low loss coupling is essential for optical performance, as high loss coupling means that the source has to be operated high above threshold to yield the required launched power into the optical fibre. Running laser at higher driving current results in an increase of heat to be dissipated and impacts its long-term stability and reliability. The excitation of a flat-end optical fibre by a Gaussian beam has been studied by several authors [3-5].

\section{Power Coupling Efficiency}

The power carried by the Gaussian beam using a full-wave analysis based on the formulation of electric and magnetic components as [6] shown in the Figure 1 is derived by

$$
P_{s}=\frac{1}{2} R_{E} \int_{-\infty}^{\infty} d x \int_{-\infty}^{\infty} E_{i x}(x, y, 0) H_{i y}^{*}(x, y, 0) d y
$$

where $R_{E}$ represents the real part. $E_{i x}$ is the $x$ component of transverse electric field of incident Gaussian beam, $H_{i y}$ is the $y$ component of transverse magnetic field of incident Gaussian beam at $z=0$ obtained from the Hertz vector of magnetic type. The power coupled into the multimode 


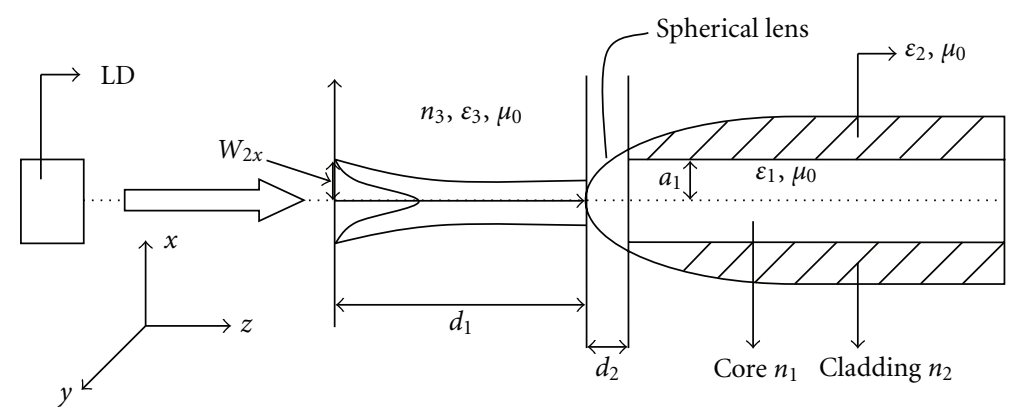

FIGURE 1: Schematic diagram of laser diode to optical fibre coupling configuration via microlens.

fibre can be obtained using the transverse components of transmitted fields in the fibre-core medium as a measure of cross-polarization as shown in the Figure 1 is given by

$$
\begin{aligned}
P_{s}= & 2 \pi^{2}\left|E_{\omega}\right|^{2} \int_{0}^{k_{3}} d \alpha \int_{0}^{\sqrt{\left(k_{3}^{2}-\alpha_{2}\right)}}\left(\frac{\gamma^{2} \beta_{3}}{\omega \mu_{0}}+\frac{\omega \varepsilon_{3} \alpha^{2}}{\beta_{3}}\right) \\
& \times \frac{\exp \left[-(1 / 2)\left(\omega_{2 x}^{2} \alpha^{2}+\omega_{2 y}^{2} \gamma^{2}\right)\right]}{\alpha^{2}+\gamma^{2}} d \gamma,
\end{aligned}
$$

where $E_{\omega}=E_{o} \omega_{2 x} \omega_{2 y} / 2 \pi$, here $E_{o}$ is a constant field amplitude. $\alpha$ and $\beta$ are the limits of integration to take the cladding of the fibre into account in accordance with the fibre angle of acceptance. $\varepsilon_{1}, \varepsilon_{2}$, and $\varepsilon_{3}$ are the permittivities of fibre, cladding and the medium (between the source and the fibre). $\beta_{3}=\sqrt{\left(k_{3}-\alpha^{2}-\gamma^{2}\right)}$, here $k_{3}=n_{3} k_{o}=\omega \sqrt{\left(\mu_{o} \varepsilon_{3}\right)}$, and $k_{o}=2 \pi / \lambda, \lambda$ is the wavelength of the Gaussian beam. $d_{1}$ is the working distance between laser diode and lens. $d_{2}(=R)$ is the maximum depth of the lens. To prevent coupling loss due to source and fibre axial separation, the distance of separation between the source and the fibre $z_{o}$ is limited $z_{o} \leq \alpha / \tan \theta_{A}$, where $\theta_{A}=\sin ^{-1}\left[(2 \Delta)^{1 / 2} /\left(1-\Delta_{c}\right)\right]$; $\theta_{A}$ is the fibre acceptance angle. $\Delta=1-n_{2} / n_{1} ; \Delta_{c}=$ $1-n_{3} / n_{1}$. The power coupled into the fibre will then be independent of the separation $z_{o}$ when the spot size of the Gaussian beam is small compared with the core size of the fibre, $\max \left[\omega_{2 x}\left(z_{o}\right), \omega_{2 y}\left(z_{o}\right)\right] \ll a_{1}$, where $\omega_{2 x}\left(z_{o}\right)$ and $\omega_{2 y}\left(z_{o}\right)$ are the beam halfwidths along the $x$ and $y$. Integration in (1) can then be approximated by the $2 \mathrm{D}$ delta function as

$$
\begin{aligned}
& \int_{-\infty}^{+\infty} d x \int_{-\infty}^{+\infty} \exp \left[i\left(\alpha^{\prime}-\alpha\right) x+i\left(\gamma^{\prime}-\gamma\right) y\right] d y \\
& =4 \pi^{2} \delta\left(\alpha^{\prime}-\alpha\right) \delta\left(\gamma^{\prime}-\gamma\right)
\end{aligned}
$$

where $\alpha^{\prime}$ and $\gamma^{\prime}$ are relatively changed limits of integration to take the cladding of the fibre into account in accordance with the fibre angle of acceptance. Therefore, the power coupled into the fibre becomes

$$
\begin{aligned}
P_{f}= & 8 \pi^{2}\left|E_{\omega}\right|^{2} \int_{0}^{\gamma_{A}} d \alpha \int_{0}^{\gamma_{A}}\left[\frac{\gamma^{2} \beta_{3}^{2} \beta_{1}}{\omega \mu_{0}\left(\beta_{3}+\beta_{1}\right)^{2}}+\frac{\omega \varepsilon_{1} \beta_{1} \alpha^{2}}{n_{1}^{2} \beta_{3} /\left(n_{3}^{2}+\beta_{1}\right)^{2}}\right] \\
& \times \frac{\exp \left[-(1 / 2)\left(\omega_{2 x}^{2} \alpha^{2}+\omega_{2 y}^{2} \gamma^{2}\right)\right]}{\alpha^{2}+\gamma^{2}} d \gamma,
\end{aligned}
$$

where $\beta_{1}=\sqrt{\left(k_{1}-\alpha^{2}-\gamma^{2}\right)}$, here $k_{1}=n_{1} k_{o}=\omega \sqrt{\left(\mu_{0} \varepsilon_{1}\right)}$, and $k_{o}=2 \pi / \lambda$; here $n_{1}$ is the refractive index of the core, $n_{2}$ is refractive index of the cladding and $n_{3}$ is the refractive index of the medium between the source and the fibre. The power coupling efficiency of an elliptical-spot size Gaussian beam into a multimode step-index fibre is obtained using $P_{s}$ and $P_{f}$ as

$$
\begin{aligned}
\eta & =\frac{P_{f}}{P_{s}} \\
& =\frac{4 \int_{0}^{\alpha A} d \alpha \int_{0}^{\gamma A}\left[\left(\gamma^{2} \beta_{3}^{2} \beta_{1} /\left(\omega \mu_{0}\left(\beta_{3}+\beta_{1}\right)^{2}\right)\right)+\left(\omega \varepsilon_{1} \beta_{1} \alpha^{2} /\left(n_{1}{ }^{2} \beta_{3} /\left(n_{3}{ }^{2}+\beta_{1}\right)\right)^{2}\right) \times \exp \left[-(1 / 2)\left(\omega_{2 x}^{2} \alpha^{2}+\omega_{2 y}^{2} \gamma^{2}\right)\right] /\left(\alpha^{2}+\gamma^{2}\right)\right] d \gamma}{\int_{0}^{k_{3}} d \alpha \int_{0}^{\sqrt{\left(k_{3}{ }^{2}-\alpha^{2}\right)}}\left(\left(\gamma^{2} \beta_{3} / \omega \mu_{0}\right)+\left(\omega \varepsilon_{3} \alpha^{2} / \beta_{3}\right)\right)\left(\exp \left[-(1 / 2)\left(\omega^{2}{ }_{2 x} \alpha^{2}+\omega^{2}{ }_{2 y} \gamma^{2}\right)\right] /\left(\alpha^{2}+\gamma^{2}\right)\right) d \gamma} .
\end{aligned}
$$

The laser diode to the optical fibre coupling efficiency via microlens on the fibre tip is given by the well-known overlap integral [7]

$$
\eta_{x 0}=\left|\int_{s 2} \psi_{L x z} \psi_{F x o}^{*} d S_{2}\right|^{2}
$$




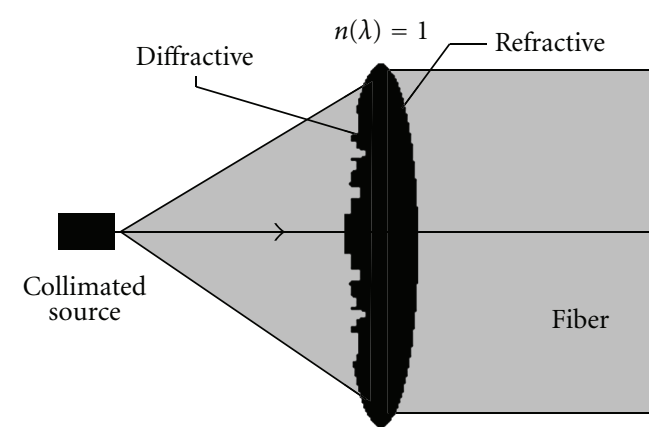

Figure 2: Axial chromatic aberration corrected hybrid lens (Refractive-Diffractive lens).

where $\psi_{L x z}$ is $x-z$ component of the light beam emitted from the laser and $\psi_{\text {Fxo }}^{*}$ is the $x-z$ component of the optical fibre field just behind the microlens at $z=0$ and $d S_{2}$ is the area of the field distributed, then the above equation becomes

$$
=4 \pi 2\left|\int_{0}^{+\infty} \psi_{L x z} \psi_{F x o}^{*} x d x\right|^{2},
$$

In the practical coupling configuration, the physical size of the microlens is limited and only part of laser light can be collected by the microlens. To take this effect into account, a microlens transmissivity factor $T_{x}$ is introduced and is given by

$$
T_{x}=2 \pi \int_{0}^{R}\left|\int_{0}^{+\infty} \psi_{L x 2}\right|^{2} x d x=1-\exp \left(-\frac{2 R^{2}}{W_{L x 2}^{2}}\right),
$$

where $R$ is the radius of the microlens. The effective coupling can be written as

$$
\eta_{x}=\eta_{x o} T_{x}
$$

A similar expression for $\eta_{y}$ can be obtained by replacing all the $x-z$ beam parameters with those for the $y-z$ beam. Total coupling efficiency from laser to a fibre can be expressed by

$$
\eta={\sqrt{\eta_{x}} \eta_{y}}
$$

under the precondition that the efficiency cannot be enhanced and it occurs only when high NA with aberration free lenses preferred to achieve maximum coupling efficiency.

\section{Hybrid (Refractive and Diffractive) Microlens}

The two microlenses can be combined in one microlens as shown in Figure 2. The advantage of such a system is that the axial chromatic aberration of the refractive component can be corrected by a diffractive component with positive focusing power owing to the negative dispersion of the diffractive optical element (DOE). In a traditional achromatic doublet, a refractive element with negative focusing power of the hybrid lens reduces the magnitude of the higher-order aberrations. The design of a hybrid achromat

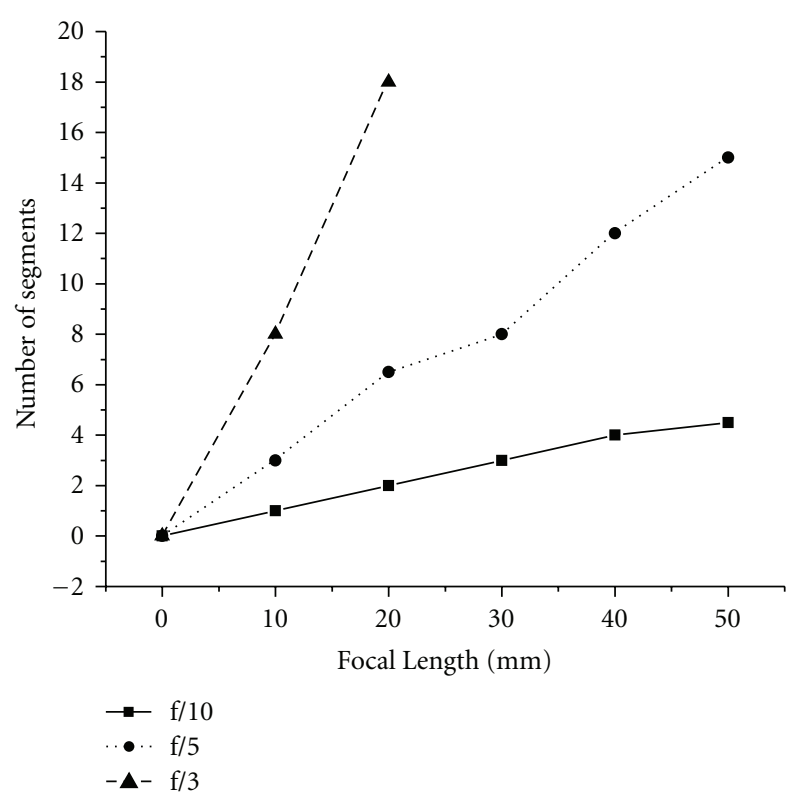

FIGURE 3: Hybrid achromat $(M=1)$ number of diffractive segments versus focal length.

is based on geometrical ray tracing. For calculating the influence of the diffractive surface, the law of refraction is replaced by the law of grating diffraction.

The focal length $f$ of a thin doublet for estimating the critical dimensions of hybrid achromat using paraxial equations is given by [8]

$$
\frac{1}{f}=\frac{1}{f_{r}}+\frac{1}{f_{d}}
$$

where in our case $f_{r}$ and $f_{d}$ are the focal lengths of the refractive and the diffractive lenses, respectively.

The condition for an achromat is then [9]

$$
f_{r} v_{r}+f_{d} v_{d}=0
$$

where the dispersions of the refractive lens and the diffractive lens are characterized by the Abbe number $v_{r}$ and $v_{d}$, respectively. For a refractive lens, the Abbe number is given by

$$
v_{r}=\frac{n_{1}-1}{n_{2}-n_{3}}
$$

where $n_{i}$ are refractive indices for the wavelengths $\lambda_{i}$. Equation (12) requires that the focal length $f$ of the achromat be the same for the wavelength is $\lambda_{1}$. The definition requires that $\lambda_{2}<\lambda_{1}<\lambda_{3}$. In the case of a diffractive lens the optical power is proportional to the wavelength; therefore, the Abbe number is given by

$$
v_{d}=\frac{\lambda_{1}}{\lambda_{2}-\lambda_{3}} .
$$

The abbe number of a refractive lens is positive, that is, $v_{r}<0$.

$$
\Delta W_{j}=M_{j} \lambda,
$$




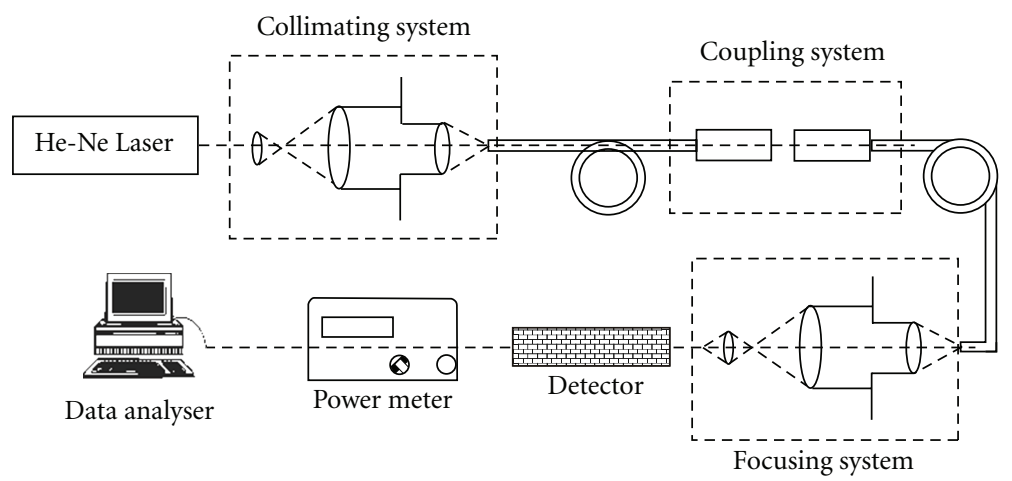

FIGURE 4: Experimental setup for the measurement of the power coupling efficiency.

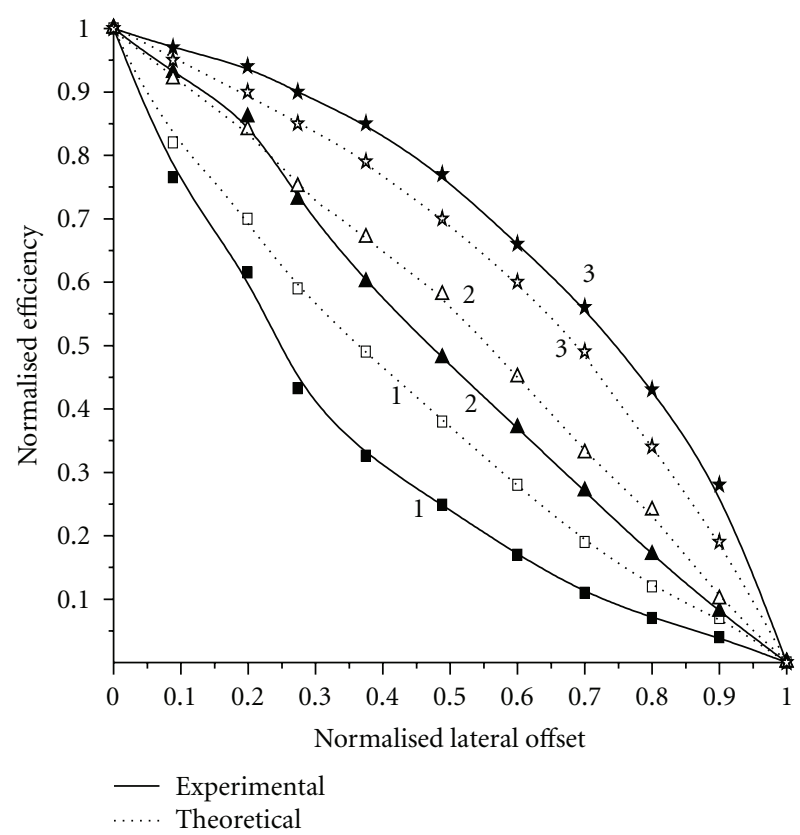

FIGURE 5: variation of coupling efficiency for (i) refractive microlens, (ii) Diffractive microlens and (iii) Hybrid microlens.

where $W_{j}$ is equivalent expression for the optical path length, $M_{j}$ is called phase-matching number, and $\lambda$ is the wavelength in air

$$
r_{j}^{2}=2 j M_{j} \lambda_{o}^{\prime} l^{\prime},
$$

where $r_{j}$ is the distance of the segment boundary from the origin having the $M_{j}$ th order focus at the position $l^{\prime}$ and $\lambda_{o}^{\prime}$ is the design wavelength in air.

From (15) and (16), we can calculate $f_{d}$ as

$$
f_{d}=f\left(1-\frac{v_{r}}{v_{d}}\right) .
$$

The phase function $\Phi(r)$ of a thin diffractive lens with focal length $f_{d}$ is described by

$$
\Phi(r)=\frac{\pi r^{2}}{\lambda_{1} f_{d}} .
$$

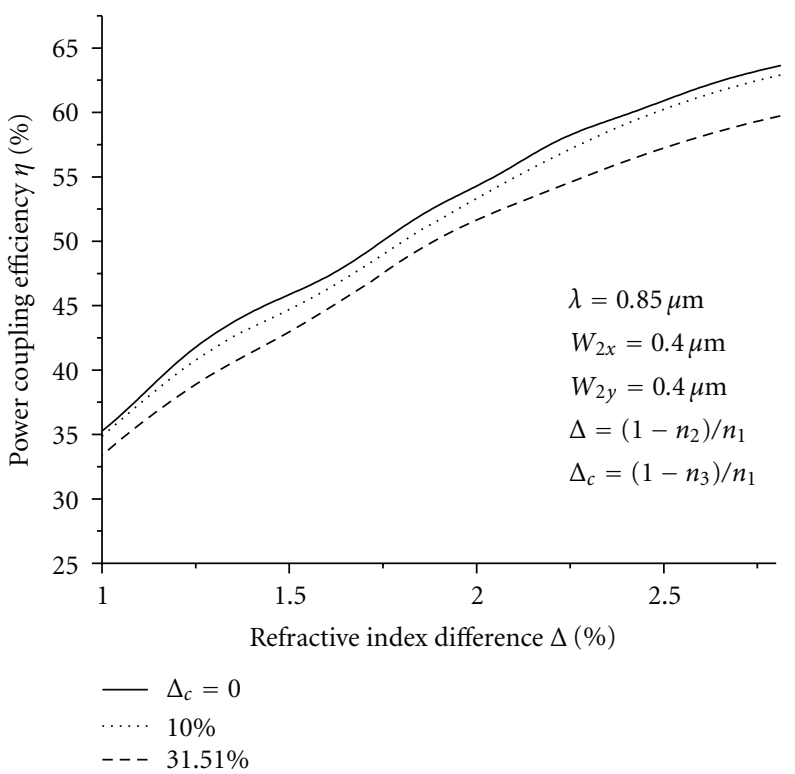

FIGURE 6: The power coupling efficiency $\eta$ is shown as a function of fibre relative core-cladding refractive index difference $\Delta$ in percent for different index mismatch $\Delta_{c}$.

For a lens of radius $a_{1}$ the number $Q$, of illuminated segments is then given by

$$
Q=\frac{\Phi(r)}{2 \pi M}=\frac{\alpha^{2}}{2 \lambda_{1} f_{d} M} .
$$

From equations $f_{d}$ and $Q$ it obtains an estimation for the number $Q$ of illuminated segments of the diffractive component. $Q$ is a function of the focal length $f$ and the $f$-number $\left(f / \#=f / 2 a_{1}\right)$ of the hybrid lens, namely,

$$
Q=\frac{f}{8 \lambda M(f / \#)^{2}}\left(\frac{v_{d}}{v_{d}-v_{r}}\right) .
$$

Figure 3 shows a graphical representation of equation $Q$. It shows that the number of illuminated segments depends on both the phase-matching number $M$ and the aperture of the element. The main reason for the low number of segments 


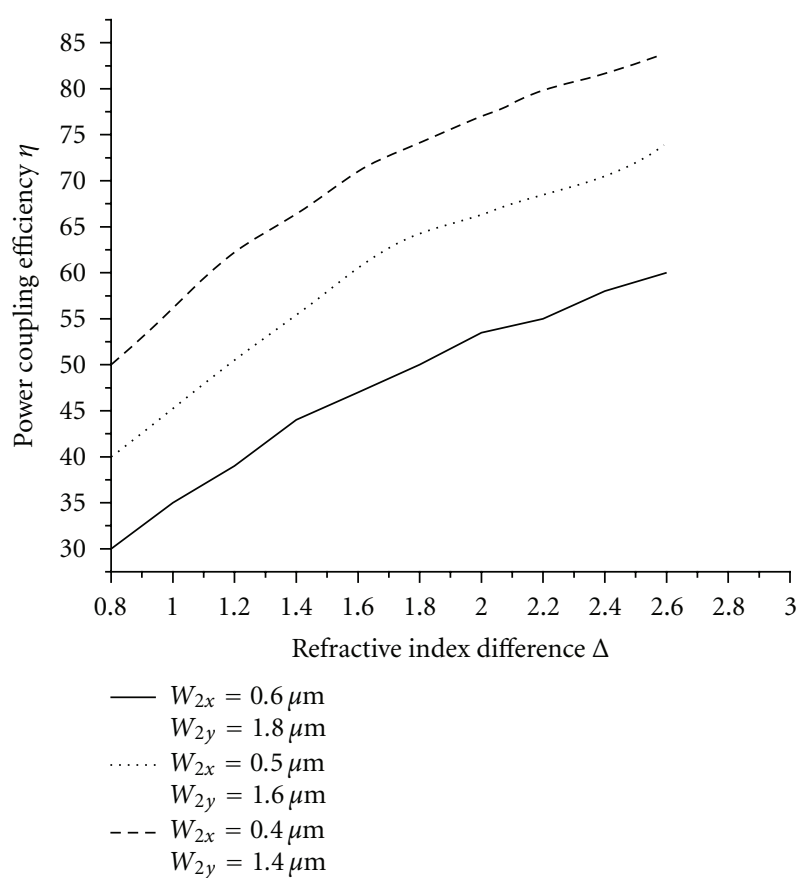

Figure 7: Power coupling efficiency $\eta$ is shown as a function of fibre relative core-cladding refractive-index difference $\Delta$ in percent for three different spot sizes fo the Gaussian beam at its waist, the wavelength of the beam, $\lambda=0.86 \mu \mathrm{m}$ and $n_{1}=1.45$.

$Q$ is that the dispersion of a refractive component is much smaller than the dispersion of a diffractive component. Owing to the results based on electromagnetic theory [10], structures designed by scalar theory would yield high efficiencies in this regime. An optimisation would need very computing intensive rigorous diffraction calculations. The slightest deviation of the experimental from the theoretical beam diameter can be explained by inaccuracies in this measurement set up. Some design rule can be given for different ranges of numerical apertures using the paraxial approximation; the number $Q$ of illuminated segments is given by

$$
Q=\frac{l^{\prime}(\mathrm{NA})^{2}}{2 M \lambda_{o}} .
$$

For low numerical apertures, elements with a constant $M_{j}$ and $M$ can be designed that have either purely refractive or purely diffractive properties. Therefore, with a suitable choice of $M$, both types of behaviour are accessible. For many technologies, the fabrication of high-NA elements is made possible only with the Phase-Matched Fresnel-Element (PMFE) concept.

\subsection{Experiments and Numerical Simulations on the Refractive-} Diffractive Behaviour. The experimental setup for the measurement of the coupling efficiency is illustrated schematically in Figure 4. An input He-Ne laser light $(\lambda=633 \mathrm{~nm})$ was coupled into a fibre, and the output end of the fibre was

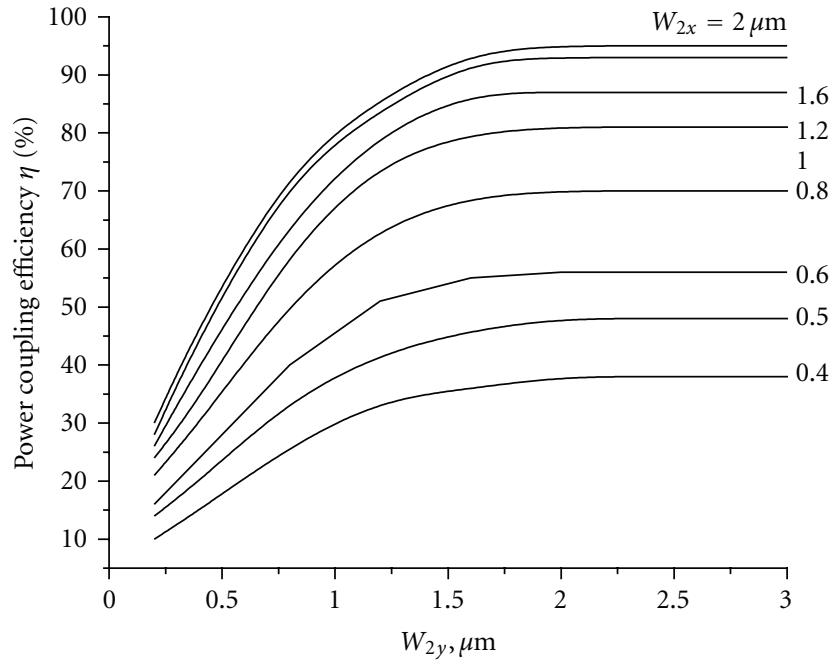

Figure 8: Power coupling efficiency $\eta$ is shown as a function of beam halfwidth $\omega_{2 x}$ at the beam waist for different beam halfwidth $\omega_{2 y}$ at the beam waist. The wave length of the Gaussian beam $0.8 \mu \mathrm{m}$, the fibre parameters are $n_{1}=1.45, \Delta=1 \%$ and $\mathrm{NA}=0.25$.

joined to the input end of the coupled Refractive-diffractive microlenses. The fibre is set in contact with the front surface, and its near field pattern is transformed as an inverted image at the back surface. The transformed near field pattern was observed using detector, power meter, and data analyser. Collimating and focussing systems had improved the characteristics of the modified output field profile to focus into a fibre [11]. The variation of coupling efficiency of microlenses due to the lateral offset of normalised coupling efficiency for (i) refractive microlenses (ii) diffractive microlenses, and (iii) refractive-diffractive microlenses (hybrid microlenses) is shown in the Figure 5. Computed coupling efficiency was in good agreement with the experimental ones.

\section{Result and Discussion}

Based on the full-wave analysis and the numerical results, several important observations can be made. Equation (5) is calculated numerically using 10-point Gauss-Legendre quadrature [12] for $\gamma$ integration and $\alpha$ integration with five subintervals in $\gamma$ and $\alpha$ between their respective integration limits resulting in a four-digit accuracy of $\eta$.

For fibre of core radius $a_{1}=50 \mu \mathrm{m}$ with an air gap between the optical source and the fibre, $\Delta_{c}=31.51 \%$, according to the source-fibre separation $z_{o}$ is limited to $z_{o}<$ $238 \mu \mathrm{m}$ for $\Delta=1 \%$ and to $z_{0}<135 \mu \mathrm{m}$ for $\Delta=3 \%$. Consider $\Delta_{c}$ as a measure of index mismatch for power coupling into the fibre. The wavelength of the Gaussian beam is $\lambda=$ $0.85 \mu \mathrm{m}$, and the spot size at its waist is $\omega_{2 x}=0.4 \mu \mathrm{m}, \omega_{2 y}=$ $1.2 \mu \mathrm{m}$. The refractive index of fibre core is $n_{1}=1.46$. The power coupling efficiency $\eta$ is shown in Figure 6 as a function of the fibre relative core-cladding refractive $\Delta$ at different index mismatch $\Delta_{c}=0,10 \%, 31.5 \%$. The case of a perfect index match is indicated by $\Delta_{c}=0$, and $\Delta_{c}=31.5 \%$ indicates 


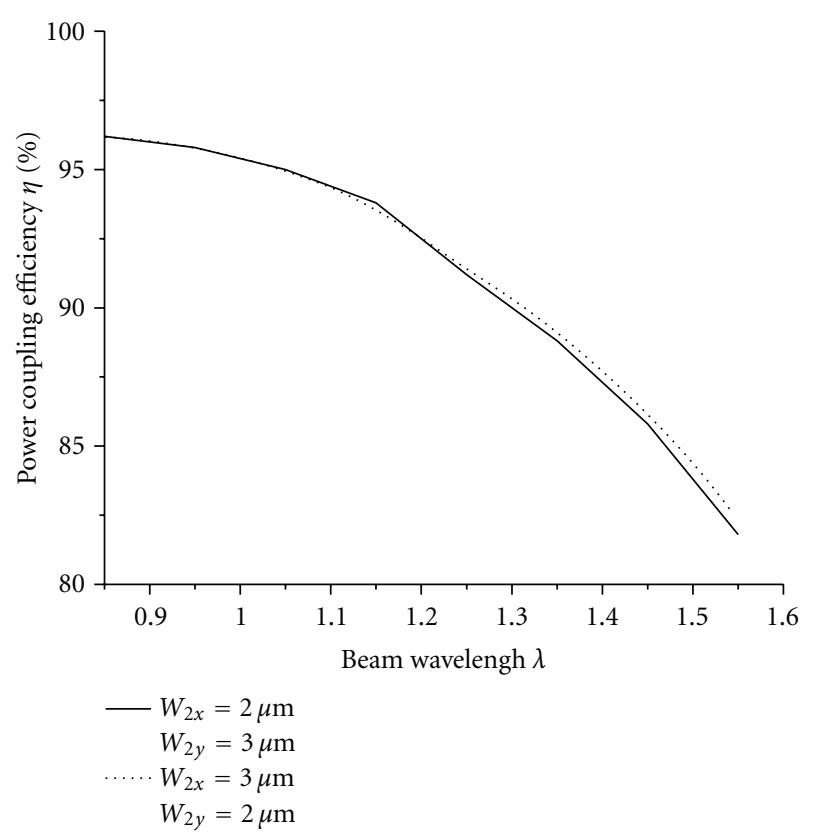

FIgURe 9: Power coupling efficiency $\eta$ is shown as a function of beam wavelength $\lambda$ for two different beam spot sizes at the beam waist. The fibre parameters are $n_{1}=1.45, \Delta=1 \%$ and NA $=0.25$.

that the medium $\varepsilon_{3}=$ is air or free space. Figure 7 shows that the power coupling efficiency for a perfect index match is not significantly different from that for index mismatch by $\Delta_{c}=10 \%$. For typical case of air gap between the optical source and the fibre with index mismatch by $\Delta_{c}=31.5 \%$, the power coupling efficiently decreases from $2.87 \%$ for a fibre $\Delta=1 \%$ to $4.71 \%$ for a fibre of $\Delta=2.8 \%$ when compared with the case of perfect index match. It is indicated that the coupling efficiency increases with increasing fibre $\Delta$.

The spot size of the Gaussian beam at the beam waist affects the power coupling efficiency; $\eta$ is shown in Figure 8 as a function of the beam halfwidth $\omega_{2 x}$ at the beam waist for the following different beam halfwidths $\omega_{2 x}$ at the beam waist, $\omega_{2 x}=0.4,0.5,0.6,0.8,1.0,1.2,1.6,2.0 \mu \mathrm{m}$. The wavelength of the Gaussian beam is $\lambda=0.84 \mu \mathrm{m}$, the fibre parameters are $n_{1}=1.45, \Delta=1 \%$, and N.A $=0.205$. From the Figure 8, when either $\omega_{2 x}$ or $\omega_{2 y}$ is $<1.6 \mu \mathrm{m}, \eta$ decreases rapidly with decreasing beam spot size at the beam waist. When $\omega_{2 x}$ or $\omega_{2 y}$ is $>1.6 \mu \mathrm{m}, \eta$ increases slowly with increasing beam spot size at the beam waist and $\eta$ approaches a maximum value limited by the smallest beamwidth either $\omega_{2 x}$ or $\omega_{2 y}$ at the beam waist. When both $\omega_{2 x}$ and $\omega_{2 y}$ at the beam waist are $>1.6 \mu \mathrm{m}, \eta$ increases slowly and approaches a value of $96.34 \%$ at $\omega_{2 x}=2.0 \mu \mathrm{m}, \omega_{2 y}=3.0 \mu \mathrm{m}$. This is still less than the power transmission coefficient of a perpendicular incident plane wave, $T=4 n_{1} /\left(n_{1}+1\right)^{2}=$ $96.63 \%$. From Figure 8, we found that the power coupling efficiency $\eta$ increases not only with increasing $\omega_{2 y}$ but also with increasing $\omega_{2 x}$ to an approximate saturation situation $\omega_{2 x} \geq 2 \mu \mathrm{m}, \omega_{2 y} \geq 3 \mu \mathrm{m}$. These occur because the beam halfwidth [13] $\omega_{2 x}\left(z_{0}\right)$ at any point $z_{0}$ is a function not only of $z_{o}$ but also of wavelength $\lambda$ and the beam halfwidth at the beam waist $\omega_{2 j}(0)$

$$
\omega_{2 x}\left(z_{o}\right)=\omega_{2 j}\left(z_{o}\right)\left(1+\frac{\lambda^{2} z_{o}^{2}}{\pi^{2} \omega_{2 j}{ }^{4}(0)}\right)^{1 / 2}, \quad j=x, y .
$$

In Figure 9, the power coupling efficiency is shown as a function of Gaussian beam wavelength $\lambda$ with the fibre parameters $n_{1}=1.45, \Delta=1 \%$, N.A. $=0.205$ for two different beam spot sizes $\omega_{2 x}=2.0 \mu \mathrm{m}, \omega_{2 y}=3.0 \mu \mathrm{m}$ at the beam waist. It shows that the power coupling efficiency decreases with increasing wavelength because the spread of the Gaussian beam with increasing wavelength according to (22) results in a coupling loss. The power coupling efficiency $\eta$ is slightly higher for the spot size $\omega_{2 x}=2.0 \mu \mathrm{m}, \omega_{2 y}=$ $3.0 \mu \mathrm{m}$ at the beam waist for $\lambda \geq 0.92 \mu \mathrm{m}$. expressed in terms of different directions of the electric polarization at the beam waist. This difference in coupling efficiency increases slightly with increasing wavelength to only $0.48 \%$ at $\lambda=1.60 \mu \mathrm{m}$. However, the power coupling efficiency is slightly lower for the spot size for $\omega_{2 x}=3.0 \mu \mathrm{m}, \omega_{2 y}=2.0 \mu \mathrm{m}$ at the beam waist than for the spot size $\omega_{2 x}=2.0 \mu \mathrm{m}, \omega_{2 y}=3.0 \mu \mathrm{m}$ at the beam waist for $\lambda<0.92 \mu \mathrm{m}$. This difference is very small only $0.01 \%$ at $\lambda=0.86 \mu \mathrm{m}$.

The following numerical simulations are performed on a series of eight different (PMFE) concepts, which focus on collimated He-Ne laser beam at a distance $l^{\prime}=4.0 \mathrm{~mm}$. These lenses have a numerical aperture NA $=0.05(f / \#=$ 10); their phase-matching numbers are varied from $M=1$ to $M=8$. The PMFE with $M=1$ consists of $Q=8$ segments, and because of the $2 \pi$ phase steps, it represents the purely diffractive case [14]. Owing to the clamped finite aperture, the case of a purely refractive microlens is reached by the elements with $M=8$. According to the increasing size and the decreasing number of segments boundaries, the elements with phase-matching numbers in the range from $M=2$ to $M=7$ can be described as having both refractive and diffractive behaviour. Coupling loss is reduced with microlens used as coupler even increasing the wavelength of the Gaussian beam when ignoring the interference patterns obtained due to diffractive elements.

\section{Conclusion}

The power coupling efficiency of an elliptical size Gaussian beam into a multimode step-index fiber via microlens is derived using a full-wave analysis. It is found that the power coupling efficiency increases with decreasing index mismatch. The power coupling loss due to beam spread is very large when the spot size at the beam waist is small. To obtain a higher power coupling efficiency, the Gaussian beam spot size at the beam waist should be larger, but is still small compared with the core size of the fiber. There is a slight difference in power coupling efficiency for a large spot size due to the different direction of the electric polarization at the beam waist. This difference increases slightly with increasing wavelength of the Gaussian beam. The power coupling loss caused by beam spread is also large when the wavelength of the Gaussian beam is increased. To obtain 
higher power coupling efficiency operating at a longer spot size at its waist and the spot size should be still small compared with the core size of the fiber. The Gaussian beam is not a pure transverse wave. The transmitted beam has changed the electric polarization in the fiber-core medium.

By variation of the number of segments at a constant numerical aperture, the transition between purely refractive and diffractive lenses is numerically simulated. Based on these results, design rules for power coupling efficiency including low- and high-numerical aperture lenses and hybrid refractive-diffractive elements are investigated. For a high-numerical aperture lens, the experimental characterisation of the irradiance distribution in the image space (detector) is presented and shown to agree well with theoretical predications.

\section{Acknowledgments}

The authors is very thankful to Dr. K. G. Ravikumar, Fujikura Ltd., Japan, for both his continuous encouragement and hospitality. S. Selvanadan would like to thank Professor S. Mohan, Raman School of Physics, Pondicherry University, Pondicherry and Dr. P.M. Anbarasan for the use of laboratory and software facilities. The author is also deeply indebted to the anonymous reviewers for their constructive and helpful comments.

\section{References}

[1] J. -M. Park, S. -G. Lee, H. -R. Park, and M. -H. Lee, "High-efficiency polarization beam splitter based on a selfcollimating photonic crystal," Journal of the Optical Society of America B, vol. 27, no. 11, pp. 2247-2254, 2010.

[2] W. H. Wee and J. B. Pendry, "Shrinking optical devices," New Journal of Physics, vol. 11, Article ID 073033, 2009.

[3] D. Marcuse, "Radiation losses of the dominant mode in round dielectric wave guides," Bell System Technical Journal, vol. 49, p. $1690,1971$.

[4] Imai and E. H. Hara, "Excitation of fundamental and loworder modes of optical fiber waveguides by gaussian beams. 1: tilted beams," Applied Optics, vol. 13, no. 8, pp. 1893-1899, 1974.

[5] Imai and E. H. Hara, "Excitation of fundamental and loworder modes of optical fiber waveguides by gaussian beams. 1: tilted beams," Applied Optics, vol. 14, p. 169, 1975.

[6] J. T. Horng and D. C. Chang, "Coupling an elliptical Gaussian beam into a multimode step-index fibre," Applied Optics, vol. 22, no. 23, pp. 3887-3891, 1983.

[7] J. John, T. S.M. Maclean, H. Ghafouri-Shiraz, and J. Niblett, "Matching of single-mode fibre to laser diode by microlenses at $1.5 \mu \mathrm{m}$ wavelength," IEE Proceedings: Optoelectronics, vol. 141, no. 3, pp. 178-184, 1994.

[8] W. T. Welford, Aberrations of Optical Systems, chapter 11.3 and chapter 10.2, Hilger, London, UK, 1986.

[9] E. Hecht and A. Zajar, Optics, chapter 6.3, Addison-Wesley, Reading, Mass, USA, 1987.

[10] E. Noponen, J. Turunen, and A. Vasara, "Electromagnetic theory and design of diffractive-lens arrays," Journal of the Optical Society of America A, vol. 10, no. 3, pp. 434-443, 1993.

[11] P. M. Anbarasan and S. Mohan, "Theoretical and experimental study on couplihg property of distributed-index microlenses n micro-optics," Journal of Optics, vol. 33, no. 1, pp. 37-45, 2004.

[12] M. Abramowitz and I. A. Stegun, Handbook of Mathematical Functions, Dover, New York, NY, USA, 1964.

[13] D. Marcuse, Light Transmission Optics, Van Nostrand Reinhold, New York, NY, USA, 1982.

[14] K. Miyamoto, “The phase Fresnel lens," Journal of the Optical Society of America, vol. 51, pp. 17-20, 1961. 

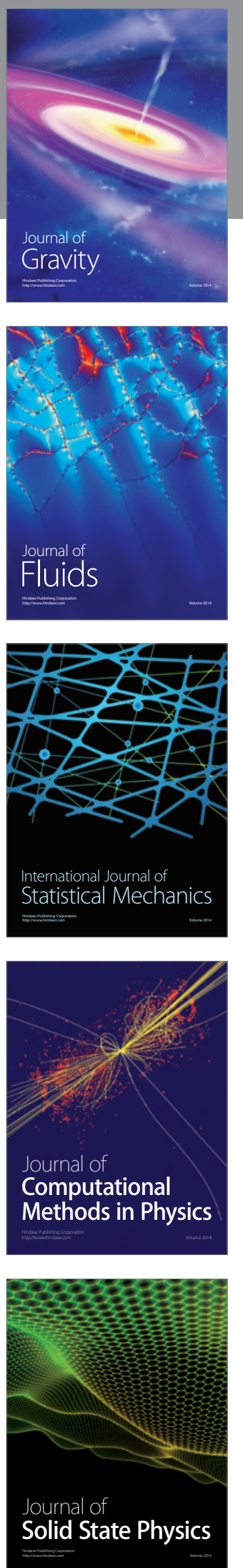

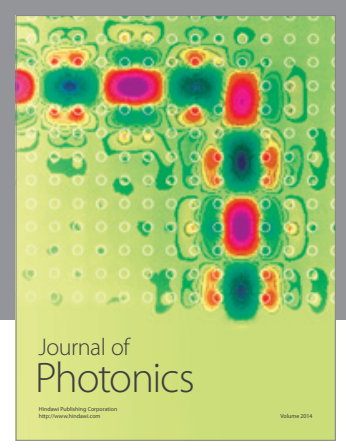

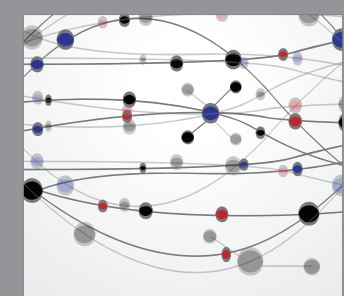

The Scientific World Journal
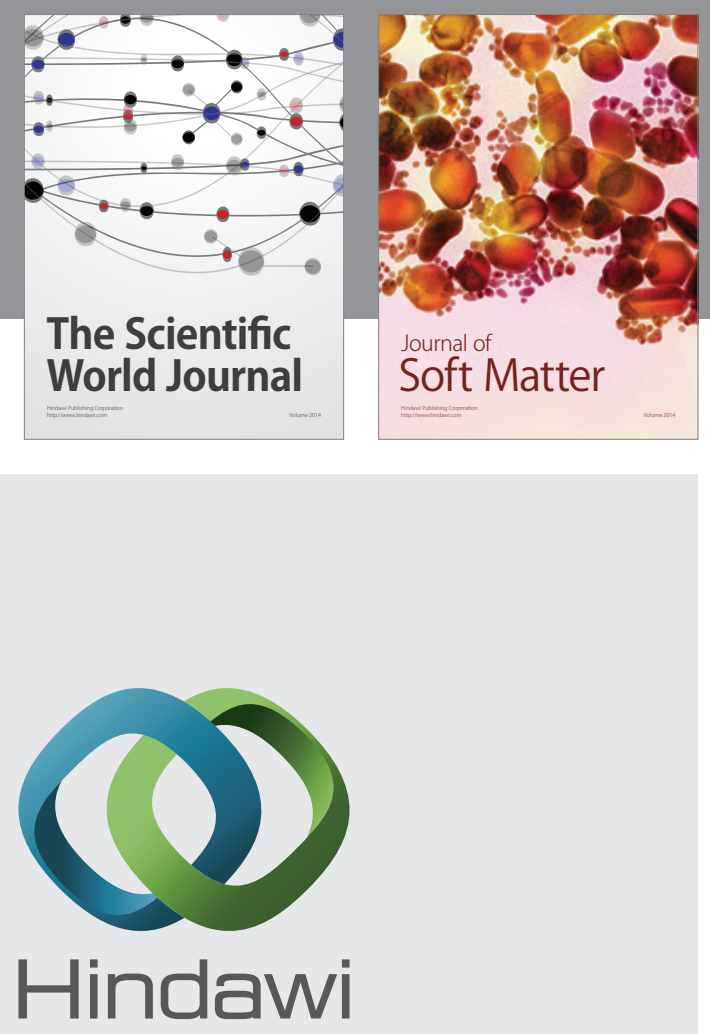

Submit your manuscripts at

http://www.hindawi.com
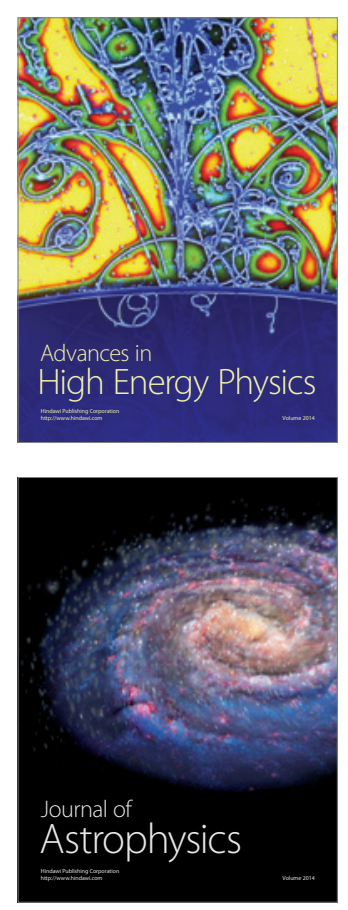
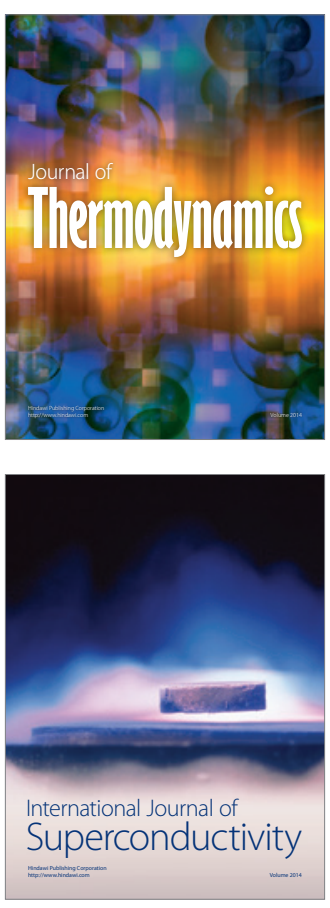
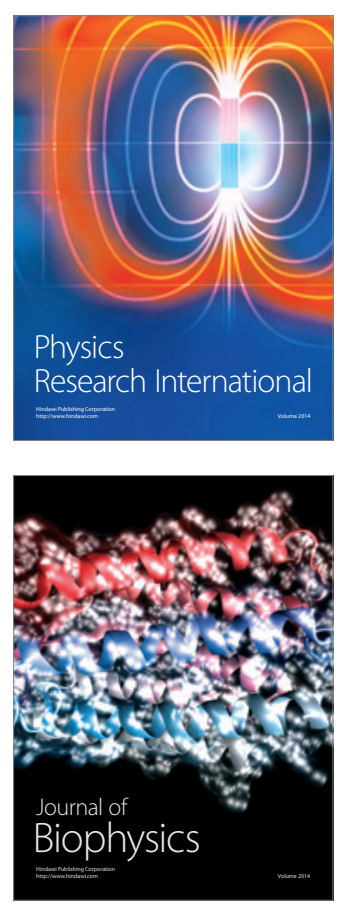
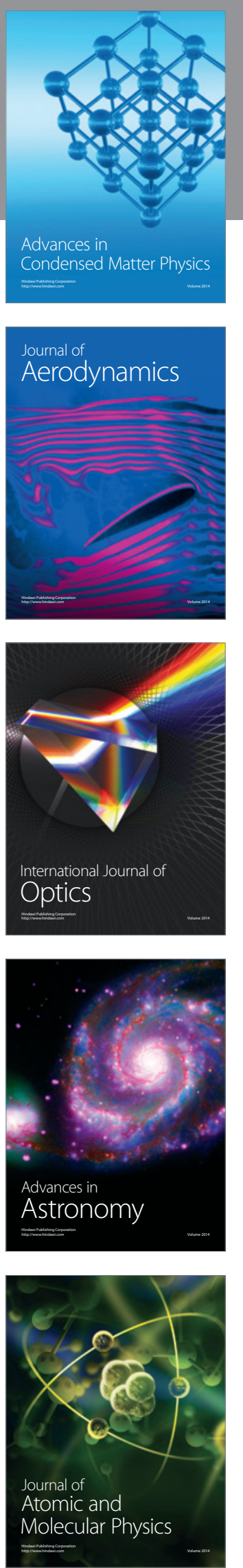